Char act erized di vertor foot print prof i l e modi f i cat i on wi th the edge pressure gradi ent in the Large Hel i cal Devi ce

\begin{tabular}{|l|l|}
\hline $\begin{array}{l}\text { jour nal or } \\
\text { publ i cat i on } \mathrm{titl} \text { e }\end{array}$ & $\mathrm{Pl}$ asma Physi cs and Cont rol I ed Fusi on \\
\hline vol une & 60 \\
\hline number & 12 \\
\hline page range & 125001 \\
\hline year & $2018-10-12$ \\
\hline URL & ht t p: //hdl . handl e. net /10655/00012935 \\
\hline
\end{tabular}




\title{
Characterized divertor footprint profile modification with the edge pressure gradient in the Large Helical Device
}

\author{
H. Tanaka ${ }^{1}$, S. Masuzaki ${ }^{2}$, G. Kawamura ${ }^{2,3}$, M. Kobayashi ${ }^{2,3}$, Y. Suzuki ${ }^{2,3}$, G. Motojima ${ }^{2,3}$, \\ T. Murase $^{2}$, T. Morisaki ${ }^{2,3}$, N. Ohno ${ }^{1}$, and LHD Experiment Group ${ }^{2}$ \\ ${ }^{1}$ Graduate School of Engineering, Nagoya University, Chikusa-ku, Nagoya 464-8603, Japan \\ ${ }^{2}$ National Institute for Fusion Science, National Institutes of Natural Sciences, Toki, Gifu 509- \\ 5292, Japan \\ ${ }^{3}$ SOKENDAI (Graduate University for Advanced Studies), Toki 509-5292, Japan
}

E-mail: h-tanaka@ees.nagoya-u.ac.jp

\begin{abstract}
The first attempt to characterize the divertor footprint profile in the heliotron device LHD was done, by using a number of Langmuir probes and the multivariable analysis technique. In order to clarify the generation mechanism of the private-side peak on the footprint profile, which has not been reproduced in the modeling study, over 6000 time points were extracted by excluding time points with profile modifications due to already-known reasons. A characterization index $r_{2 / 1}$ was newly defined from the multivariable analysis result, and its dependences on upstream parameters were investigated. As a result, it was found that the footprint profile correlates with the pressure gradient at the edge inside the core region with a fixed beta, suggesting that change of the plasma pressure profile could modify the edge magnetic field structure even if the volume integral of the plasma pressure was constant.
\end{abstract}

Keywords: divertor footprint profile, Langmuir probe, multivariable analysis, proper orthogonal decomposition, LHD

PACS: 52.25.Xz, 52.55.Hc, 52.70.Ds, 02.70.Rr

\section{Introduction}

Clarification of the shaping mechanism of the divertor footprint profile across the strike point is important for the accurate prediction of divertor heat and particle loads in future fusion devices. In tokamaks, footprint profiles are fitted and characterized with an expression which is derived from the convolution of an exponential decay and a Gaussian function [1]. In heliotron/stellarator devices, however, a similar approach is difficult mainly owing to the non-simple three-dimensional (3D) magnetic field structure around the divertor plate: for example, connection length of the magnetic field has several peaks across the strike point, like tokamaks with a strong resonant magnetic perturbation (RMP) field [2]. Establishment of the characterization procedure of such a complicated footprint 
profile is quite essential in order to proceed with the clarification of the parameter dependence and to perform the multi-machine comparison.

In the heliotron device LHD, divertor configuration is generated naturally by twisted helical coils without a divertor coil [3]. It is known that the footprint profile is mainly determined by the vacuum magnetic field depending on typical magnetic field parameters such as magnetic axis position $R_{\mathrm{ax}}$, quadruple magnetic field $B_{\mathrm{q}}$, and pitch parameter $\gamma$. In addition, plasma current against the toroidal magnetic field strength $\left(I_{\mathrm{p}} / B_{\mathrm{ax}}\right)$ induces the footprint profile shift due to the change of the rotation-transform profile, just like a result from the $\gamma$ change [4]. Furthermore, high averaged beta $(\langle\beta\rangle)$ condition of $\langle\beta\rangle>\sim 1 \%$ moves the divertor-flux concentration along the helical direction, which generates similar effect to that resulting from the $R_{\mathrm{ax}}$ increase [5]. The above-described dependences are experimentally and theoretically understood. However, there is an insufficiently understood footprint modification depending on the edge electron temperature $\left(T_{\mathrm{e}, \text { edge }}\right)$. In the experiment, private-side peak appears on the divertor flux profile when $T_{\mathrm{e}, \text { edge }}$ is high, and it disappears when $T_{\mathrm{e}, \text { edge }}$ is low [6]. In the numerical modeling, however, such experimentally observed private-side peak has not been reproduced by using the 3D transport code EMC3-EIRENE even with introducing spatially non-uniform cross-field transport coefficients [7]. This modeling result suggests that the private-side peak is possibly generated by the high-energy particle or the change of the equilibrium magnetic field structure due to the local current flowing somewhere. Further, the experimental result in Ref. [6] is obtained from only one discharge. Therefore, statistical approach from dozens or a few hundred of discharges is required.

In this study, the divertor footprint profile of the ion particle flux from all discharges in the 17th experimental campaign was characterized with standard magnetic field parameters $\left(R_{\mathrm{ax}}=3.6 \mathrm{~m}, B_{\mathrm{q}}=100 \%\right.$, and $\left.\gamma=1.254\right)$ without high $\left|I_{\mathrm{p}} / B_{\mathrm{ax}}\right|$ and $\langle\beta\rangle$ in LHD. To characterize the footprint profile, a multivariable analysis method called the proper orthogonal decomposition (POD) was introduced. By using the POD output, complicated footprint profiles were successfully characterized with a normalized index, $r_{2 / 1}$. After the characterization with $r_{2 / 1}$, upstream parameter dependences of $r_{2 / 1}$ were investigated. As a result, it was found that appearance of the private-side peak depends on the edge electron density in addition to the edge electron temperature. Furthermore, change of the edge pressure gradient was found to modify the footprint profile even if $\langle\beta\rangle$ was constant. Obtained results suggest that the divertor footprint profile and the edge magnetic field structure would be modified due to the Pfirsch-Schlüter current driven by the pressure gradient even if the volume integral of the plasma pressure was the same.

In the following, experimental setup and the construction of the dataset will be described. Next, the POD method will be explained and applied in Sec. 3. In Sec. 4, characterization of the footprint profile will be performed from the POD output. Then, upstream parameter dependences will be investigated in Sec. 5. Finally, this study will be summarized in Sec. 6 .

\section{Experimental setup and making a dataset}

The LHD has two superconducting coils twisting with a toroidal period number of $m=10$. Double-null like poloidal cross section rotates poloidally by changing the toroidal angle. Thus, divertor plates terminating four divertor legs also rotate in the helical direction. In contrast to the double-null tokamak configuration, core plasma 
is surrounded by the ergodic region consisting of stochastic magnetic field lines with long connection length. This means that the magnetic field lines connecting to the divertor plate with high heat and particle fluxes rotate numerous times around the core without making the flux surfaces. In the tokamak device, the divertor footprint is mainly determined by the upstream plasma condition at the low-field side scrape-off layer (SOL) except for the detached divertor case; the mapping on the low-field side midplane provides a meaningful result and is used for the multi-machine scaling [1]. However, in LHD, there is no unique point representing the upstream plasma along a long magnetic field line due to the existence of the ergodic region. This makes the tokamak-like approach more difficult in addition to the $3 \mathrm{D}$ nature.

In the inward-shifted magnetic axis condition $\left(R_{\mathrm{ax}}=3.6 \mathrm{~m}\right)$, which is the standard magnetic field configuration achieving high-performance plasmas in LHD, most divertor flux concentrates to the radially innerside divertor plates [8]. Figure 1(a) shows the 3D profile of vacuum magnetic field connection length $\left(L_{\mathrm{c}}\right)$ calculated at the vessel wall radius for $R_{\mathrm{ax}}=3.6 \mathrm{~m}$ configuration. It is seen that the thick long- $L_{\mathrm{c}}$ area is localized at the radially inner-side of the torus. Furthermore, inside the inner-side torus, the thick area positions at the upperleft and lower-right regions viewed from the radially outer-side position [9], as shown in the magnified view in Fig. 1(a). On 14 divertor plates positioning near the inner-side midplane in 7 toroidal sections, a total of 280 Langmuir probe electrodes were installed. We call them as "toroidal divertor probe arrays" [10]. The toroidal divertor probe arrays detect the typical peak flux along the helical direction in each toroidal section under the $R_{\mathrm{ax}}$ $\sim 3.6 \mathrm{~m}$ condition.

In this study, a large part of the toroidal divertor probe arrays with a total of 200 probes, which are positioned more precisely on helically symmetric 10 divertor plates in 5 toroidal sections, were investigated. Figure 1(b) shows locations of the analyzed divertor plates in the LHD top view. In each toroidal section (\#2, 6, 7, 8, and 10), two probe arrays are located at left- (L) and right- $(\mathrm{R})$ hand sides of the private region which are viewed from the radially outer-side positions, as shown in Fig. 1(c). The top view in Fig. 1(b) also shows positions of plasma heating systems of the neutral beam injection devices with negative (N-NBI) and positive ion sources (P-NBI), the electron cyclotron heating (ECH) mirrors, and the ion cyclotron heating (ICH) antennas. N-NBI and P-NBI inputted 180 $\mathrm{keV}$ and $40 \mathrm{keV}$ hydrogen beams in tangential and perpendicular directions, respectively. Figure 1(d) shows the $L_{\mathrm{c}}$ profile along a divertor probe array for $R_{\mathrm{ax}}=3.6 \mathrm{~m}$ configuration. Each plate has 20 probe tips, and they align across the strike point with several $L_{\mathrm{c}}$ peaks from the private side to the SOL side every $6 \mathrm{~mm}$. The private-side peak is observed at probe channels 5-8 in Fig. 1(d). Figure 1(e) shows the 2D $L_{\mathrm{c}}$ profile on the poloidal cross section with a $\mathrm{L}$ plate. It is found that the peeled magnetic field lines from the ergodic region, which looks like the lobe structure [11], make the long- $L_{\mathrm{c}}$ region thick. The toroidal divertor probe arrays were installed from the 16th experimental campaign in 2012 and are being operated at present through slight modifications.

In this study, ion saturation current $\left(I_{\mathrm{sat}}\right)$ signals, which is proportional to the ion fluxes, acquired in the 17 th experimental campaign in 2013 were analyzed. Firstly, $200 I_{\text {sat }}$ signals in all discharges (a total of 7,384 discharges) were collected from 3 to $7 \mathrm{~s}$ every $0.1 \mathrm{~s}$ in each discharge. Here, $0 \mathrm{~s}$ is the start timing of an experimental sequence in a discharge, and 3-7 s covers a large part of discharge periods. After that, we selected standard inward-shifted magnetic configuration: $R_{\mathrm{ax}}=3.6 \mathrm{~m}, B_{\mathrm{q}}=100 \%, \gamma=1.254$, and sufficiently small RMP field. Moreover, to remove the high- $\langle\beta\rangle$ effect, strong toroidal magnetic field condition $\left(B_{\mathrm{t}}=-2.75 \mathrm{~T}\right)$ was selected. In addition, plasma- 
current effect was removed by selecting time points which fulfill $\left|I_{\mathrm{p}} / B_{\mathrm{ax}}\right|<10 \mathrm{kA} / \mathrm{T}$, corresponding to the $L_{\mathrm{c}}$-peak shift of $<\sim 2.5 \mathrm{~mm}$ in the past study [4]. Furthermore, time points with upstream plasma and without some measurement troubles were extracted. Finally, 6409 time points in 276 discharges were extracted from the 17th experimental campaign. Approximately $98 \%$ of extracted time points are $\langle\beta\rangle<1 \%$, which were measured with the diamagnetic loop. Approximately $66 \%$ of extracted time points are heated by only the NBI. Heating powers of N-NBI and P-NBI were less than $\sim 14 \mathrm{MW}$ and $\sim 8.5 \mathrm{MW}$, respectively. Discharge gas was basically hydrogen, and the impurity injection was not distinguished.

Figure 2 shows the 2D pattern of $I_{\text {sat }}$ as functions of the probe channels in 10 divertor plates $(x)$ and extracted time points $(t)$. In this figure, from left- to right-hand sides, $I_{\text {sat }}$ at $2 \mathrm{~L}, 2 \mathrm{R}, 6 \mathrm{~L}, 6 \mathrm{R}, 7 \mathrm{~L}, 7 \mathrm{R}, 8 \mathrm{~L}, 8 \mathrm{R}, 10 \mathrm{~L}$, and $10 \mathrm{R}$ plates are jointed. In the experiment, resistances connecting to 12 probe channels were frequently broken due to the electrical insulation deterioration of the probe tip, which are not plotted in Fig. 2. These channels were removed for the analysis below. Therefore, the $188 \times 6409$ matrix was analyzed.

\section{Proper orthogonal decomposition of the 2D dataset}

In order to characterize the divertor particle flux profile, the 2D dataset obtained in the previous section was analyzed by using the POD technique that does not utilize any artificial orthogonal function unlike the Fourier expansion [12]. The POD method is also called the principal component analysis (PCA), the empirical orthogonal function (EOF), or the Karhunen-Loéve (K-L) expansion. This method is often applied for the analysis of multidimensional data obtained by the fast imaging camera and so on. By using the POD method, typical profile patterns and their appearance time points can be extracted from a large dataset.

In this method, multipoint fluctuations $\tilde{I}(x, t) \equiv I(x, t)-\langle I(x, t)\rangle$ at position $x$ are expanded as

$$
\tilde{I}(x, t)=\sum_{i=1}^{n} a_{i}(t) \phi_{i}(x),
$$

where $a_{i}(t)$ and $\phi_{i}(x)$ are the orthogonal basis for a time domain and the orthonormal basis for a space domain, respectively. Here, \langle\rangle means an average in a time. In order to decompose $\tilde{I}(x, t)$, the covariance matrix $\mathbf{C}$ was calculated by

$$
\mathbf{C}=\left(\begin{array}{cccc}
C_{11} & C_{12} & \cdots & C_{1 n} \\
C_{21} & C_{22} & \cdots & C_{2 n} \\
\vdots & \vdots & \ddots & \vdots \\
C_{n 1} & C_{n 2} & \cdots & C_{n n}
\end{array}\right),
$$

which consists of the cross-correlation function $C_{l m}$ between positions $x_{l}$ and $x_{m}$ defined by

$$
C_{l m}=C\left(I\left(x_{l}\right), I\left(x_{m}\right)\right) \equiv\left\langle\tilde{I}\left(x_{l}, t\right) \tilde{I}\left(x_{m}, t\right)\right\rangle .
$$

Normalized cross-correlation function defined by $R_{l m} \equiv C_{l m} /\left\langle\tilde{I}^{2}\left(x_{l}, t\right)\right\rangle\left\langle\tilde{I}^{2}\left(x_{m}, t\right)\right\rangle$, which is called the crosscorrelation coefficient, will be also used in between the other parameters in below. After the calculation of $\mathbf{C}$, an eigenvalue problem with $\mathbf{C}$ is solved as

$$
\mathbf{C} \phi_{i}(x)=\lambda_{i} \phi_{i}(x),
$$

where $\phi_{i}(x)$ and $\lambda_{i}$ are the eigenfunction and the eigenvalue, respectively. Norm of $\phi_{i}(x)$ is one, and subscript $i$ is descending order of magnitude of $\lambda_{i}$ as $\lambda_{1}>\lambda_{2}>\cdots>\lambda_{n}$. Basis for a time domain $a_{i}(t)$ is calculated from

$$
a_{i}(t)=\sum_{k=1}^{n} \tilde{I}\left(x_{k}, t\right) \phi_{i}\left(x_{k}\right),
$$


and $\left\langle a_{i}^{2}(t)\right\rangle=\lambda_{i}$. Therefore, ratio of $\lambda_{i}$ to sum of $\lambda_{i}, \lambda_{i} / \sum_{k=1}^{n} \lambda_{k}$, indicates the contribution ratio for the variance in all space domain.

In this study, the offset-added $a_{i}(t)$ was newly introduced as

$$
a_{i}^{\prime}(t) \equiv a_{i}(t)+\sum_{k=1}^{n}\left\langle I\left(x_{k}, t\right)\right\rangle \phi_{i}\left(x_{k}\right),
$$

in order to make $a_{i}^{\prime}(t)=0$ when $I(x, t)=0$, which means plasma does not exist.

Figure 3(a) shows calculated $\lambda_{i} / \sum_{k=1}^{n} \lambda_{k}$ from the 2D pattern in Fig. 2 as a function of $i$. It is noted that $i=$ 1 and 2 are approximately $72 \%$ and $15 \%$, respectively, meaning that $a_{1} \phi_{1}$ dominantly determines the $I_{\text {sat }}$ fluctuations, and $87 \%$ of the fluctuations can be reconstructed from $a_{1} \phi_{1}+a_{2} \phi_{2}$. Therefore, all $I_{\text {sat }}$ profiles can be roughly reconstructed from $a_{1}^{\prime} \phi_{1}+a_{2}^{\prime} \phi_{2}$. Figure 3(b) shows $\phi_{1}$ and $\phi_{2}$ profiles as a function of the probe channel on each divertor plate. At the all probe channels, $\phi_{1}$ has no negative value. We can see that there are positive peaks at private-side and SOL-side probe channels in $\phi_{1}$. Contrastingly, $\phi_{2}$ has negative and positive peaks at the private-side and SOL-side of each footprint profile, respectively. These profiles resemble each other in all divertor plates, although amplitudes of $\phi_{1}$ and $\phi_{2}$ on R plates are larger than those on L plates. This amplitude difference between R and L plates is attributed to $\boldsymbol{E} \times \boldsymbol{B}_{\mathrm{t}}$ and/or grad- $\boldsymbol{B}_{\mathrm{t}}$ drift transports, because the magnitude relationship switches when $B_{\mathrm{t}}$ direction changes, as reported in the past study [5]. $\phi_{i}$ with $i \geq 3$ components contain more fine variations and toroidally asymmetric features, which is attributed to the localized gas puffing [9] and so on. Because this study used a number of helically symmetric divertor plates, toroidally localized effects become small in the POD output.

Because $\lambda_{1} \gg \lambda_{2}$ and $\left|\sum_{L+R} \phi_{2}\right| /\left|\sum_{L+R} \phi_{1}\right|$ is small $(\sim 0.15)$, summation of $I_{\text {sat }}$ on all divertor plates, $\sum_{L+R} I_{\text {sat }}$, is mainly determined by $a_{1}^{\prime}$. Figure $3(\mathrm{c})$ is a scatter plot of $a_{1}^{\prime}$ versus $\sum_{L+R} I_{\text {sat }}$, and the dot color indicates $a_{2}^{\prime}$ value. Strong linear relationship between $\sum_{L+R} I_{\text {sat }}$ and $a_{1}^{\prime}$ is clearly seen; dispersion due to the $a_{2}^{\prime}$ variance is much smaller. Therefore, $a_{1}^{\prime}$ indicates summed $I_{\text {sat }}$ on the divertor footprint. On the other hand, as mentioned in the next section, $a_{2}^{\prime}$ contains an information in relation to the peak position of the footprint profile.

Ratio between summation of $\phi_{1}$ on $\mathrm{L}$ plates $\left(\sum_{L} \phi_{1}\right)$ and that on R plates $\left(\sum_{R} \phi_{1}\right), \sum_{L} \phi_{1}: \sum_{R} \phi_{1}$, is approximately $1: 1.5$. Also, the same analysis for a total of 4326 time points in 187 discharges with $B_{\mathrm{t}}=+2.75 \mathrm{~T}$ was performed. As a result, $\sum_{L} \phi_{1}: \sum_{R} \phi_{1} \sim 1.8: 1$. Thus, averaged ratio for $B_{\mathrm{t}}<0$ and $B_{\mathrm{t}}>0$ cases is roughly 1: 1.65 , being equal to $0.75: 1.25$, on smaller and larger plates. Therefore, $\sim 25 \%$ of the divertor particle flux was averagely transferred from $\mathrm{L} / \mathrm{R}$ to $\mathrm{R} / \mathrm{L}$ plates for $B_{\mathrm{t}}<0 / B_{\mathrm{t}}>0$ case by the drift transport in the analyzed discharges. Difference between the above-estimated ratios for $B_{\mathrm{t}}<0$ and $B_{\mathrm{t}}>0$ cases could be attributed to the slight difference of the plate angle against the magnetic field and the difference of the averaged plasma parameters in the two cases. In tokamaks, such transport effect is observed as the $B_{\mathrm{t}}$-direction dependence of the in-out asymmetric divertor flux [13].

\section{Characterization of $I_{\text {sat }}$ profiles from the POD output}

In this section, the $I_{\text {sat }}$ profile is characterized with the POD analysis result. Figure 4 shows an example of time trends of several parameters in a discharge (shot number: \#121523) with dramatic changes of the $I_{\text {sat }}$ profile. As shown in Fig. 4(a), ramp-up of the plasma is done by ECH; then, subsequent N-NBI keeps the plasma for several seconds. During the discharge, hydrogen gas puff and tungsten (W) pellet injection are performed. After 
W-pellet injection, $P_{\text {rad }}$ rapidly increases and then decreases. Figure 4(b) shows a contour plot of $I_{\text {sat }}$ on $8 \mathrm{R}$ plate. Interestingly, private-side two peaks and SOL-side peak alternately appear. This $I_{\text {sat }}$ pattern is a portion of the analyzed 2D pattern, as depicted with the label “\#121523 8R" in Fig. 2. Figure 4(c) shows the time series of $a_{1}^{\prime}$ and $a_{2}^{\prime}$. It is confirmed that $a_{1}^{\prime} \geq 0$ and $a_{1}^{\prime}$ reflects the change of summed $I_{\text {sat }}$ : e.g., both $a_{1}^{\prime}$ and summed $I_{\text {sat }}$ decrease after the W-pellet injection and the drop of the NBI input power. In comparison with $a_{1}^{\prime}$, $\left|a_{2}^{\prime}\right|$ has smaller amplitude. Sign of $a_{2}^{\prime}$ alternately changes with the change of the $I_{\text {sat }}$ profile: private-side and SOL-side peaks accompany negative and positive $a_{2}^{\prime}$, respectively. Therefore, $a_{2}^{\prime}$ correlates with the peak position of the footprint profile.

The footprint profile of the particle flux will be characterized by using the POD output. Figure 5(a) is a scatter plot of $a_{2}^{\prime}$ versus $a_{1}^{\prime}$. Outer shape of data points resembles a left triangle, because basically $a_{1}^{\prime} \geq 0$ and $a_{1}^{\prime} \phi_{1}+$ $a_{2}^{\prime} \phi_{2} \approx I_{\text {sat }} \geq 0$ in all probe channels. If $a_{2}^{\prime}$ was quite large positively/negatively in comparison with $a_{1}^{\prime}$, private/SOL-side $I_{\text {sat }}$ would become negative. Here, we would like to introduce the normalized characterization index $r_{2 / 1}$ as

$$
r_{2 / 1}(t) \equiv a_{2}^{\prime}(t) / a_{1}^{\prime}(t)
$$

which corresponds to the slope from the origin in Fig. 5(a). By normalizing $a_{2}^{\prime}(t)$ with $a_{1}^{\prime}(t), r_{2 / 1}(t)$ does not have an amplitude information but characterizes the footprint profile with private-side or SOL-side peak. $r_{2 / 1}(t)$ was calculated under the condition of $a_{1}^{\prime}(t)>0.05$, because $r_{2 / 1}(t)$ with small $a_{1}^{\prime}(t)$ is easily disturbed by noise components in $I_{\text {sat. }}$. Figure 5(b) shows histogram of $r_{2 / 1}(t)$. Large part of $\left|r_{2 / 1}(t)\right|$ is less than $\sim 0.5$, as also seen in Fig. 5(a). Figure 6 shows reconstructed footprint profiles on $8 \mathrm{R}$ plate from $a_{1}^{\prime} \phi_{1}+a_{2}^{\prime} \phi_{2}$ with $r_{2 / 1}=$ -0.5 and +0.5 . If $r_{2 / 1}=0, a_{1}^{\prime} \phi_{1}+a_{2}^{\prime} \phi_{2}$ is proportional to $\phi_{1}$ in Fig. 3(b) because $a_{2}^{\prime}=0$. It can be found that the private-side peak stands out with negative $r_{2 / 1}$ in Fig. 6.

Time trend of the above-defined $r_{2 / 1}$ in a discharge is shown in Fig. 4(c). Change of $r_{2 / 1}$ from positive to negative seems to be slower than that from negative to positive. Such gradual and sudden changes are also seen in the 2D pattern of $I_{\text {sat }}$ in Fig. 4(b); thus, $r_{2 / 1}$ successfully characterizes the footprint profile. Figures 4(d), (e), and (f) indicate time trends of upstream parameters. Dependences of $r_{2 / 1}$ on them will be investigated in the next section.

\section{Upstream parameter dependences of the characterization index}

In the beginning, the cross-correlation coefficients were investigated between the characterization index $r_{2 / 1}$ and upstream parameters of the electron temperature $T_{\mathrm{e}}\left(R\left(r_{2 / 1}, T_{\mathrm{e}}\right)\right)$, the electron density $n_{\mathrm{e}}\left(R\left(r_{2 / 1}, n_{\mathrm{e}}\right)\right)$, and the electron pressure $n_{\mathrm{e}} T_{\mathrm{e}}\left(R\left(r_{2 / 1}, n_{\mathrm{e}} T_{\mathrm{e}}\right)\right)$, measured with the Thomson scattering, as shown in Fig. 7(a). Horizontal axis indicates the major radius $R$ at the midplane on the horizontally elongated poloidal cross section. Amplitude of the correlation coefficient becomes small when large noise components exist; thus, time points with small error ratios of $T_{\mathrm{e}}$ and $n_{\mathrm{e}}$ that satisfy $\Delta T_{\mathrm{e}} / T_{\mathrm{e}}<10 \%$ and $\Delta n_{\mathrm{e}} / n_{\mathrm{e}}<10 \%$ were only used. In addition, some upstream data that has clearly discontinuous $\Delta T_{\mathrm{e}}$ or $\Delta n_{\mathrm{e}}$ along $R$ or $t$ were manually and numerically removed. Figure 7(b) shows the number of remaining time points (thin solid line) as a function of $R$. Because an analysis from fewer data could be easily affected by noise components, correlation values outside the last closed flux surface (LCFS) have lower reliability. Figure 7(c) shows mean $\Delta T_{\mathrm{e}} / T_{\mathrm{e}}$ and $\Delta n_{\mathrm{e}} / n_{\mathrm{e}}$ at analyzed time points, indicating that they are almost 
constant except for around and outside the LCFS.

From Fig. 7(a), it is found that $R\left(r_{2 / 1}, T_{\mathrm{e}}\right)$ is negatively large in wide $R$ range. Near the outer-side LCFS, $R\left(r_{2 / 1}, T_{\mathrm{e}}\right) \sim-0.7$, being stronger than that around the magnetic axis position $(R=3.6 \mathrm{~m})$; in contrast, $\left|R\left(r_{2 / 1}, T_{\mathrm{e}}\right)\right|$ near the inner-side LCFS drops to $\sim 0$ from the core. Profile of $R\left(r_{2 / 1}, n_{\mathrm{e}} T_{\mathrm{e}}\right)$ is similar to that of $R\left(r_{2 / 1}, T_{\mathrm{e}}\right)$ and its amplitude has maximum at the normalized small radius of $\rho \sim 0.85$. Here, $\rho$ is a calculated value on a fixed pressure profile condition $\left(\alpha\left(1-\rho^{8}\right)\left(1-\rho^{2}\right)\right.$ with $\left.\langle\beta\rangle=0.22 \%\right)$. On the other hand, $R\left(r_{2 / 1}, n_{\mathrm{e}}\right)$ is $\sim 0$ inside the LCFS $(|\rho| \leq 1)$. Figures 4(d) and (e) display time trends of $T_{\mathrm{e}}$ and $n_{\mathrm{e}}$, respectively, at $\rho \sim 1$ and 0.85 in the sample discharge. Clear negative correlation can be seen between $r_{2 / 1}$ and $T_{\mathrm{e}}$ compared with $n_{\mathrm{e}}$. Both $T_{\mathrm{e}}$ at $\rho \sim 1$ and 0.85 change with similar time trends except for their amplitudes.

From the above analysis, edge $T_{\mathrm{e}}$ is confirmed to be an important parameter for varying $r_{2 / 1}$, as pointed out in the previous study [6], which showed a dependence on $T_{\mathrm{e}}$ at $\rho \sim 1$. Thus, we plotted $r_{2 / 1}$ versus $T_{\mathrm{e}}$ at $\rho \sim 1$ with small measurement errors satisfying $\Delta T_{\mathrm{e}} / T_{\mathrm{e}}<10 \%$ and $\Delta n_{\mathrm{e}} / n_{\mathrm{e}}<10 \%$, as shown in Fig. 8(a). Surely there is a negative correlation between $r_{2 / 1}$ and $T_{\mathrm{e}}$; however, large dispersion exists. In Sec. 5 below, additional and alternative parameters determining the footprint profile will be surveyed in terms of heating operation, parameter position, $n_{\mathrm{e}},\langle\beta\rangle$, and pressure gradient in sequence.

\subsection{Heating operation dependence}

To investigate the reason for the dispersion in Fig. 8(a), dots with only N-NBI (blue dots) and P-NBI (red dots) are superimposed by different colors at first. In this figure, a large number of red dots with only P-NBI seem to be out of alignment with blue dots with only N-NBI, suggesting that energetic ion losses from the NBI could be a possible reason for the footprint profile change. Because trajectories of the energetic ions from N-NBI strongly depend on the NB injection direction against the toroidal magnetic field direction [14], the scatter plot with only co- and ctr-injected N-NBs are additionally investigated, as shown in Fig. 8(b). It is found that there is no significant separation between them. Therefore, high-energy ion losses would not be a cause for the footprint profile modification. Actually, spatial patterns of $\phi_{1}$ and $\phi_{2}$ are toroidally almost the same (see Fig. 3(b)), suggesting that there is no relationship with the locality of the NBI position, depicted in Fig. 1(a).

\subsection{Parameter position and $n_{\mathrm{e}}$ dependences}

Next, the maximum $R\left(r_{2 / 1}, n_{\mathrm{e}} T_{\mathrm{e}}\right)$ position of $\rho \sim 0.85$ will be focused. Figure 7(c) is a similar plot to Fig. 8(a) with the exception of $\rho$. At $\rho \sim 0.85$, separation with only N-NBI and P-NBI becomes slightly smaller, although a large dispersion remains. Figure 8(d) shows a similar plot but the dots are colored by $n_{\mathrm{e}}$ at $\rho \sim 0.85$. Here, $n_{\mathrm{e}}$ amplitude is calibrated with the far infrared interferometer measurement. We can find clear color gradation in the $r_{2 / 1}-T_{\mathrm{e}}$ plot, indicating that $n_{\mathrm{e}}$ also affects the $r_{2 / 1}$ change. Separation between with only N-NBI and PNBI is not seen in this figure.

Figure 8(e) is a scatter plot of $n_{\mathrm{e}}$ versus $T_{\mathrm{e}}$ at $\rho \sim 0.85$ colored by $r_{2 / 1}$. From this figure, it is found that high $T_{\mathrm{e}}$ and high $n_{\mathrm{e}}$ contribute to the reduction of $r_{2 / 1}$, corresponding to becoming the footprint profile with the privateside peak. An existence of the $n_{\mathrm{e}}$ dependence seems to disagree with the decorrelation in Fig. 7(a). This small correlation with $n_{\mathrm{e}}$ would be caused by the co-existing dependence of $r_{2 / 1}$ on $T_{\mathrm{e}}$. For example, when the P-NBI 
located near the vacuum vessel with a large conductance was injected, the neutral gas was injected as well as the heating power. In such a case, both $n_{\mathrm{e}}$ and $T_{\mathrm{e}}$ increased, and thus $r_{2 / 1}$ decreased. On the other hand, after a simple gas puffing, $n_{\mathrm{e}}$ increased and $T_{\mathrm{e}}$ largely decreased due to the increase of $P_{\mathrm{rad}}$. At that time, $r_{2 / 1}$ would increase despite of the $n_{\mathrm{e}}$ increase. Therefore, $n_{\mathrm{e}}$ dependence would be diminished due to the $T_{\mathrm{e}}$ variation in the correlation analysis calculated with all time points. It should be noted that an interpretation from the cross-correlation coefficient with only one parameter should be taken care. Figure $8(\mathrm{e})$ also represents constant $n_{\mathrm{e}} T_{\mathrm{e}}$ lines. Although the color gradation does not completely obey the $n_{\mathrm{e}} T_{\mathrm{e}}$, edge pressure is guessed to have a strong relationship with the footprint profile modification.

\section{3. $\langle\beta\rangle$ and pressure gradient dependences}

From Fig. 8(e), plasma pressure is supposed to be a key parameter, suggesting that $\langle\beta\rangle$, which is proportional to the volume integral of the plasma pressure, might be important even if $\langle\beta\rangle\langle\sim 1 \%$. High $\langle\beta\rangle$ over $1 \%$ is already known to change the magnetic field structure.

Figure 8(f) indicates $r_{2 / 1}$ as a function of $\langle\beta\rangle$. At $\langle\beta\rangle<\sim 0.2 \%$, dots are localized at $r_{2 / 1} \sim 0.55$, meaning with the SOL-side peak. Although there seems to be a certain correlation, large scatter is found. The other possible reason for the change of the magnetic field structure is the edge local current parallel to the magnetic field. In heliotron/stellarator devices, Pfirsch-Schlüter current is a representative parallel current that is attributed to the magnetohydrodynamics equilibrium force balance of $\boldsymbol{j} \times \boldsymbol{B}=\nabla p$ and $\nabla \cdot \boldsymbol{j}=0$, where $j$ and $p$ are the plasma current and the pressure, respectively [15]. Thus, the electron pressure gradient in the radial direction $\mathrm{d}\left(n_{\mathrm{e}} T_{\mathrm{e}}\right) / \mathrm{d} R$ was investigated, because the Pfirsch-Schlüter current is originated from the plasma pressure gradient $\nabla p$. Dots in Fig. 8(f) are colored by $\mathrm{d}\left(n_{\mathrm{e}} T_{\mathrm{e}}\right) / \mathrm{d} R$ at $\rho \sim[0.85,0.95]$ calculated from the linear function fitting. If the pressure profile was constant except for its amplitude, $\mathrm{d}\left(n_{\mathrm{e}} T_{\mathrm{e}}\right) / \mathrm{d} R$ should have monotonic dependence on $\langle\beta\rangle$. Therefore, $\langle\beta\rangle$ and $\mathrm{d}\left(n_{\mathrm{e}} T_{\mathrm{e}}\right) / \mathrm{d} R$ have non-small positive correlation, and different $\mathrm{d}\left(n_{\mathrm{e}} T_{\mathrm{e}}\right) / \mathrm{d} R$ with an arbitrary fixed $\langle\beta\rangle$ indicates different plasma pressure profile. At $\langle\beta\rangle>\sim 0.5 \%, r_{2 / 1}$ clearly depends on $\mathrm{d}\left(n_{\mathrm{e}} T_{\mathrm{e}}\right) / \mathrm{d} R$ with a fixed $\langle\beta\rangle$. Figure 9 shows $r_{2 / 1}$ as a function of $\mathrm{d}\left(n_{\mathrm{e}} T_{\mathrm{e}}\right) / \mathrm{d} R$ in the condition of $0.7 \%<$ $\langle\beta\rangle<0.8 \%$. We can see the negative correlation between $r_{2 / 1}$ and $\mathrm{d}\left(n_{\mathrm{e}} T_{\mathrm{e}}\right) / \mathrm{d} R$. The cross-correlation coefficient $R\left(r_{2 / 1}, \mathrm{~d}\left(n_{\mathrm{e}} T_{\mathrm{e}}\right) / \mathrm{d} R\right)$ is $\sim-0.7$. Similarly, at $\rho \sim[0.8,0.9]$ and $[0.9,1], R\left(r_{2 / 1}, \mathrm{~d}\left(n_{\mathrm{e}} T_{\mathrm{e}}\right) / \mathrm{d} R\right)$ are calculated as $\sim-0.55$ and $\sim-0.18$, respectively. At the left bottom $\left(\langle\beta\rangle<0.4 \%\right.$ and $\left.r_{2 / 1}<-0.25\right)$ of Fig. 8(f), dots with small gradients are observed. It is to be noted that $\sim 76 \%$ of dots in this region are time points with the helium ratios $\mathrm{He} /(\mathrm{H}+\mathrm{He})$ of over $20 \%$ due to the $\mathrm{He}$ gas injection. Therefore, although there are some few exceptions with special operations, it is confirmed that $r_{2 / 1}$ depends on the pressure gradient in the edge of the core region in a fixed $\langle\beta\rangle$ condition.

Figure 4(f) shows time trends of $\langle\beta\rangle$ and $\mathrm{d}\left(n_{\mathrm{e}} T_{\mathrm{e}}\right) / \mathrm{d} R$ at $\rho \sim[0.8,0.9]$ in a sample discharge. Rapid increases of $\mathrm{d}\left(n_{\mathrm{e}} T_{\mathrm{e}}\right) / \mathrm{d} R$ and gradual increases of $\langle\beta\rangle$ are observed when $r_{2 / 1}$ becomes negative, being the consistent result with Figs. 8(f) and 9. It is concluded that the negative $r_{2 / 1}$ would be attributed to the PfirschSchlüter current driven by the edge pressure gradient inside the core region.

\section{Summary and discussion}


The mechanism which makes the private-side peak of the divertor footprint profile was investigated in LHD. In order to archive this purpose, a number of ion particle flux signals, which were measured on 10 divertor plates installed on helically symmetric locations in the 17 th experimental campaign, were collected. To exclude footprintmodification effects from already-known reasons, time points with small $\langle\beta\rangle$ and small $\left|I_{\mathrm{p}} / B_{\mathrm{ax}}\right|$ in the standard inward-shifted magnetic field configuration $\left(R_{\mathrm{ax}}=3.6 \mathrm{~m}, B_{\mathrm{q}}=100 \%\right.$, and $\left.\gamma=1.254\right)$ were extracted. After that, the extracted $188 \times 6409$ matrix was analyzed by applying the multivariable analysis method of the proper orthogonal decomposition (POD). Analysis results indicate that the footprint profile is dominantly composed of two spatial patterns, $\phi_{1}$ and $\phi_{2}$. Their appearing ratio $r_{2 / 1}$ provides good information for the characterization of the footprint profile; $r_{2 / 1}>0$ and $r_{2 / 1}<0$ indicate SOL-side and private-side peaking, respectively. This would be the first attempt to characterize the divertor footprint profile with a few indexes in heliotron/stellarator devices. In addition, left(L)-right(R) asymmetry is found in the decomposed spatial patterns. From the ratio of summed $\phi_{1}$ on $\mathrm{L}$ and $\mathrm{R}$ plates, it is found that $\sim 25 \%$ of the ion flux is averagely moved from $\mathrm{L} / \mathrm{R}$ to $\mathrm{R} / \mathrm{L}$ plates for $B_{\mathrm{t}}<0 / B_{\mathrm{t}}>$ 0 case due to the drift transport in the analyzed discharges. The POD method is useful for not only the LHD footprint but also other various multivariable data acquired in fusion devices.

By using the characterization index $r_{2 / 1}$, upstream parameter dependences were surveyed. Firstly, clear dependence on the edge $T_{\mathrm{e}}$ is confirmed, as same as the previous study. Next, dependence of the heating system is investigated. On the $r_{2 / 1}-T_{\mathrm{e}}$ plane, no clear difference exists between with only co-injected and ctr-injected NNBs, indicating that energetic ion losses would not relate to the private-side peak of the footprint profile. At the edge region inside the LCFS $(\rho \sim 0.85)$, clear dependence of $r_{2 / 1}$ on both $T_{\mathrm{e}}$ and $n_{\mathrm{e}}$ is firstly confirmed. This result suggests that the plasma pressure and/or the pressure gradient would be important for the $r_{2 / 1}$ determination. Thus, dependences on both $\langle\beta\rangle$ and $\mathrm{d}\left(n_{\mathrm{e}} T_{\mathrm{e}}\right) / \mathrm{d} R$ are investigated, and it is found that $\mathrm{d}\left(n_{\mathrm{e}} T_{\mathrm{e}}\right) / \mathrm{d} R$ at $\rho \sim[0.85,0.95]$ correlates with $r_{2 / 1}$ on a fixed $\langle\beta\rangle$ condition. Pressure gradient in the edge inside the core could change the magnetic field structure due to the Pfirsch-Schlüter current even if $\langle\beta\rangle$ was not different, which is suggested numerically in Refs. $[15,16]$. If the lobe structure elongated from the ergodic region in Fig. 1(e) moves away from the measuring position, the SOL-side peak would become small compared with the private-side peak. Such the change would be like the measuring position shift from the L/R plate with Langmuir probes in the lower-left/upper-right direction in the magnified view in Fig. 1(a). Therefore, it is concluded that the private-side peak of the divertor footprint profile would be attributed to the magnetic field change due to the edge pressure gradient inside the core region. Considering that the summation of the ion particle flux is strongly depending not on $a_{2}^{\prime}$ but on $a_{1}^{\prime}$ (see Fig. 3(c)), decomposed bases, $a_{1} \phi_{1}$ and $a_{2} \phi_{2}$, would reflect the change of upstream plasma parameters around the LCFS and the modification of the edge magnetic field structure, respectively.

From Fig. 7, cross-correlation coefficients between $r_{2 / 1}$ and upstream parameters near the inner LCFS is weaker than those near the outer LCFS. There are several possible reasons. Finite $\langle\beta\rangle$ changes magnetic field structure to outward-shifted configuration; thus, the inner LCFS position might shift in the outer direction in a part of analyzed time points with $\langle\beta\rangle>0$. In addition, positional relationship with the analyzed divertor probes might produce the in-out asymmetric feature. The divertor probes are located at inner-side of the torus (see Fig. 1(a)), and magnetic field lines traced from the divertor probes rotate poloidally due to the strong rotation transform. Then, 
they connect to position close to the ergodic region firstly at radially outer side [9]. Therefore, analyzed divertor probe signals could be easily influenced from the magnetic field change near the outer LCFS compared with the inner LCFS.

In this study, time points with impurity gases and pellet injections were not omitted. Such special operations could cause the dispersions in the scatter plots. Additionally, ion pressure gradient is not considered. Validation in particular discharges by comparing the magnetic field calculation including the local plasma current is desirable. If the validation is successfully performed in the future, the divertor probes can be used as a type of sensor for detecting the slight modification of the upstream magnetic field structure with high spatial and temporal resolutions.

In tokamak devices, following the Eich scaling of the SOL width [1], experimental results are theoretically interpreted [17, 18], and the scaling of the limiter start-up plasmas yields to the ITER limiter optimization $[19,20]$. Although this study clarified the typical footprint pattern by using the POD method, the obtained profile would not be directly compared in between heliotron/stellarator devices, due to the significant differences of the magnetic field structure on the divertor plates, unlike the tokamak configuration. Some model that connects the obtained footprint profile to the magnetic field structure should be needed before the multi-machine comparison in the heliotron/stellarator configuration.

For a design of a future heliotron/stellarator device, 3D numerical simulation with EMC3-EIRENE [7, 21, 22 ] should have an essential role to predict the $3 \mathrm{D}$ profile of the divertor footprint. Such the edge plasma simulation code assumes a fixed magnetic field geometry from the input one. Therefore, this study, which indicated that the magnetic field structure could be modified when the edge pressure gradient is strong even if $\langle\beta\rangle\langle\sim 1 \%$, contributes to the future validation study of the EMC3-EIRENE in LHD. In addition, although this study focused on the analysis of the divertor particle flux, the divertor heat flux has more essential meaning for the engineering of future fusion devices, e.g., steady-state heat load should be below $10 \mathrm{MW} / \mathrm{m}^{2}$ on the tungsten divertor with the copper alloy cooling tube [23]. We should investigate detailed characteristics of the divertor heat flux by using Langmuir probes and an infrared camera in future.

\section{Acknowledgments}

This work was supported by KAKENHI (16H06139, 15H04236, 16K18340), NIFS Collaboration Research program (NIFS17KLPF054), and NINS program of Promoting Research by Networking among Institutions (01411702).

\section{References}

[1] Eich T et al 2013 Nucl. Fusion 53093031

[2] Jakubowski M W et al 2009 Nucl. Fusion 49095013

[3] Ohyabu N et al 1994 Nucl. Fusion 34 387-399

[4] Masuzaki S et al 2013 Nucl. Fusion 53112001

[5] Masuzaki S et al 2006 Fusion Sci. Technol. 50 361-371

[6] Masuzaki S et al 2010 Contrib. Plasma Phys. 50 629-634

[7] Dai S et al 2017 Plasma Phys. Control. Fusion 59085013 
[8] Morisaki T et al 2013 Nucl. Fusion 53063014

[9] Masuzaki S et al 2002 Nucl. Fusion 42 750-758

[10] Tanaka H et al 2017 Nucl. Mater. Energy 12 214-246

[11] Frerichs H et al 2010 Nucl. Fusion 50034004

[12] Tanaka H et al 2010 Contrib. Plasma Phys. 50 256-266

[13] Boedo J A et al 2000 Phys. Plasmas 7 1075-1078

[14] Watanabe Tet al 2006 Nucl. Fusion 46 291-305

[15] Suzuki Y et al 2010 Contrib. Plasma Phys. 50 576-581

[16] Suzuki Y et al 2017 21st International Stellarator-Heliotron Workshop (ISHW2017) (Oct. 2-6, Kyoto, Japan)

[17] Goldston R J 2012 Nucl. Fusion 52013009

[18] Halpern F D et al 2016 Plasma Phys. Control. Fusion 58084003

[19] Horacek J et al 2016 Plasma Phys. Control. Fusion 58074005

[20] Kocan M et al 2015 Nucl. Fusion 55033019

[21] Kawamura G et al 2014 Contrib. Plasma Phys. 54437

[22] Kawamura G et al 2018 Plasma Phys. Control. Fusion 60084005

[23] Pitts R A et al 2017 Nucl. Mater. Energy 12 60-74 

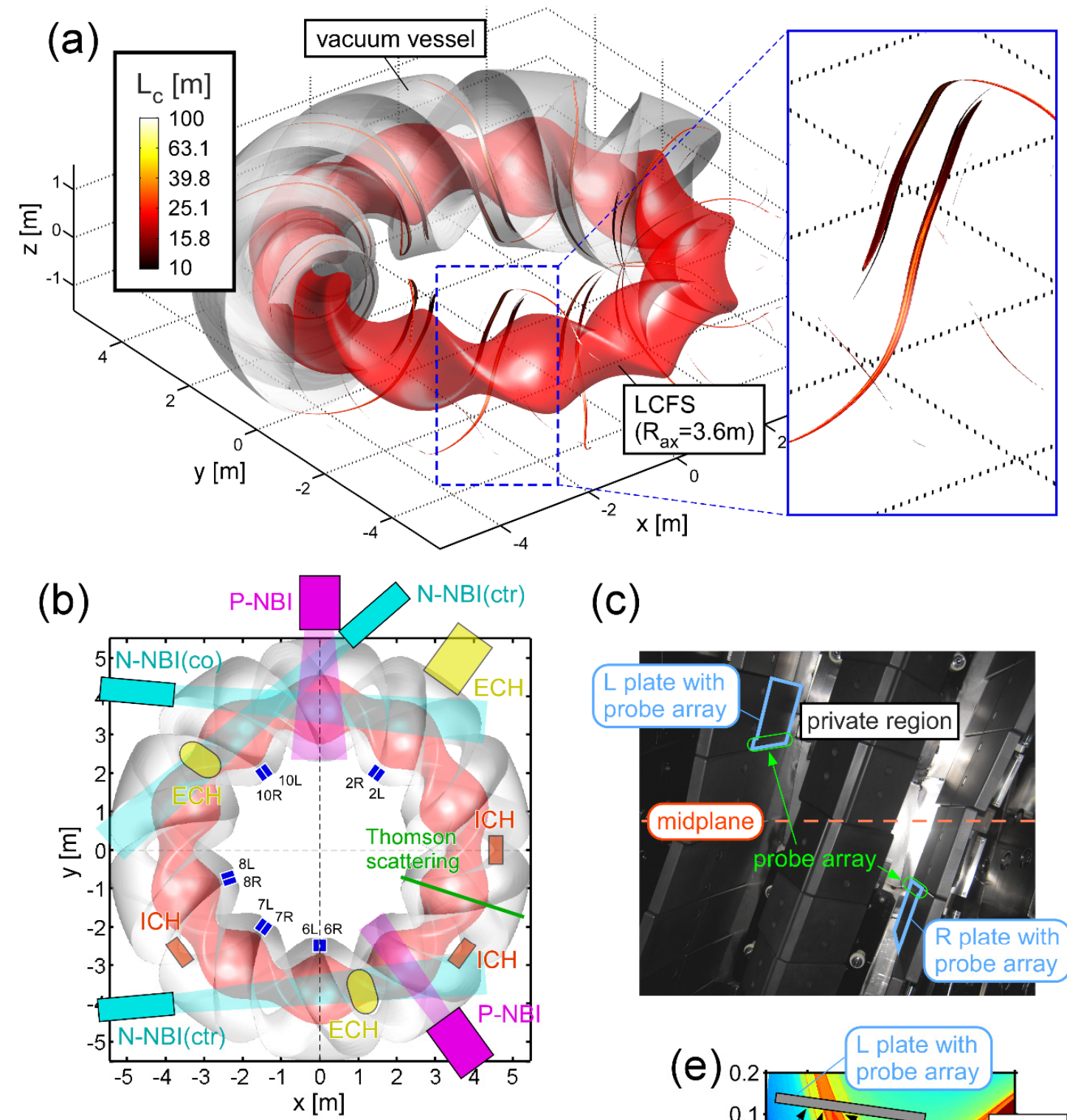

(c)
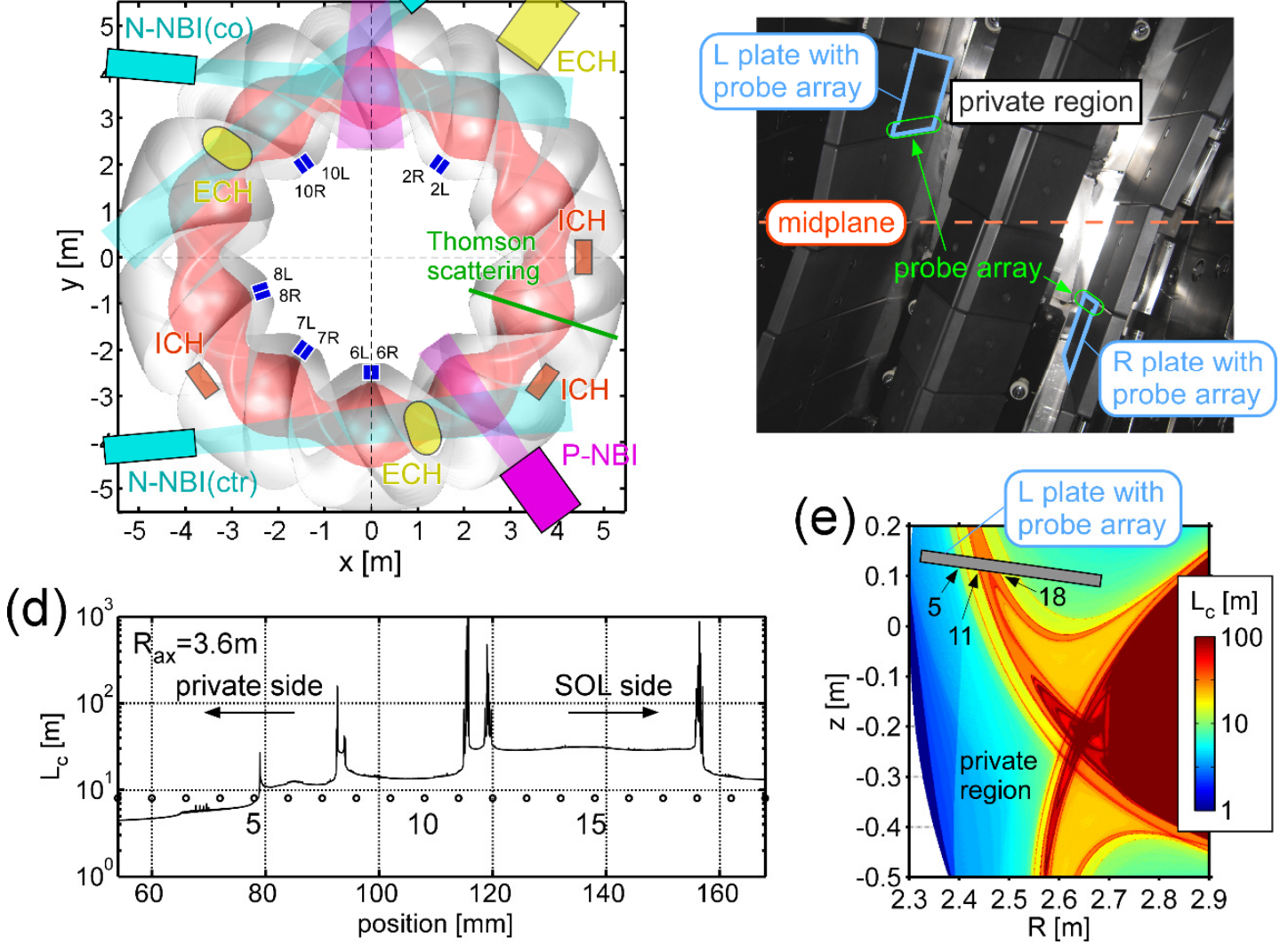

Fig. 1 (Color online) (a) 3D profile and its magnified view of $L_{\mathrm{c}}$ calculated around the vessel wall for $R_{\mathrm{ax}}=3.6 \mathrm{~m}$ configuration. (b) Top view of the toroidal divertor probe arrays and heating systems in the 17th experimental campaign. (c) Photograph of $\mathrm{L}$ and $\mathrm{R}$ plates with Langmuir probe arrays viewed from the radially outer-side position. (d) $L_{\mathrm{c}}$ profile along each divertor probe array (solid line) and the probe position (circle), where the position zero means the private-side edge of the divertor plate before the edge cutting to avoid the interference with the vessel wall during the installation. (e) $2 \mathrm{D} L_{\mathrm{c}}$ profile on the poloidal cross section with a $\mathrm{L}$ plate. 


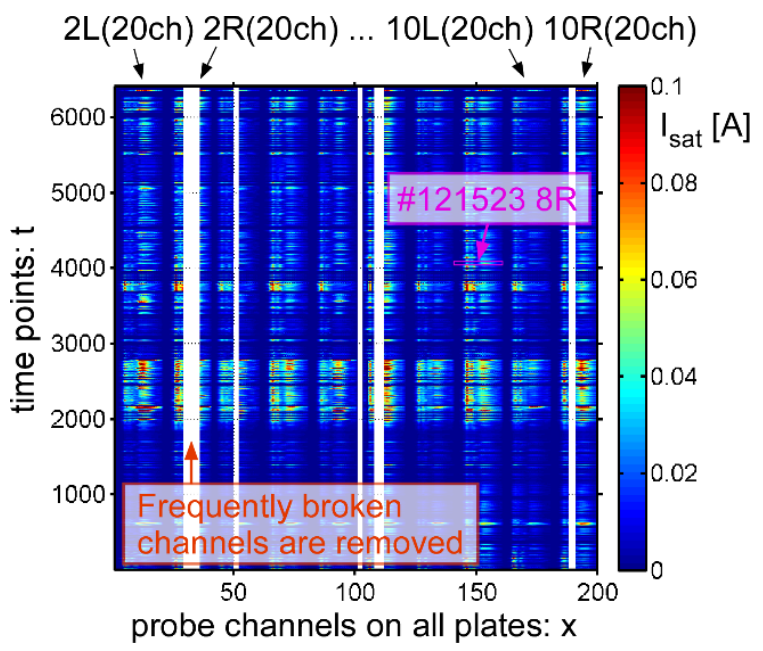

Fig. 2 (Color online) 2D pattern of $I_{\text {sat }}$ as functions of the probe channels on all analyzed divertor plates and extracted time points, where $I_{\text {sat }}$ at frequently broken channels are not plotted. A portion of this pattern corresponds to Fig. 4(b). 

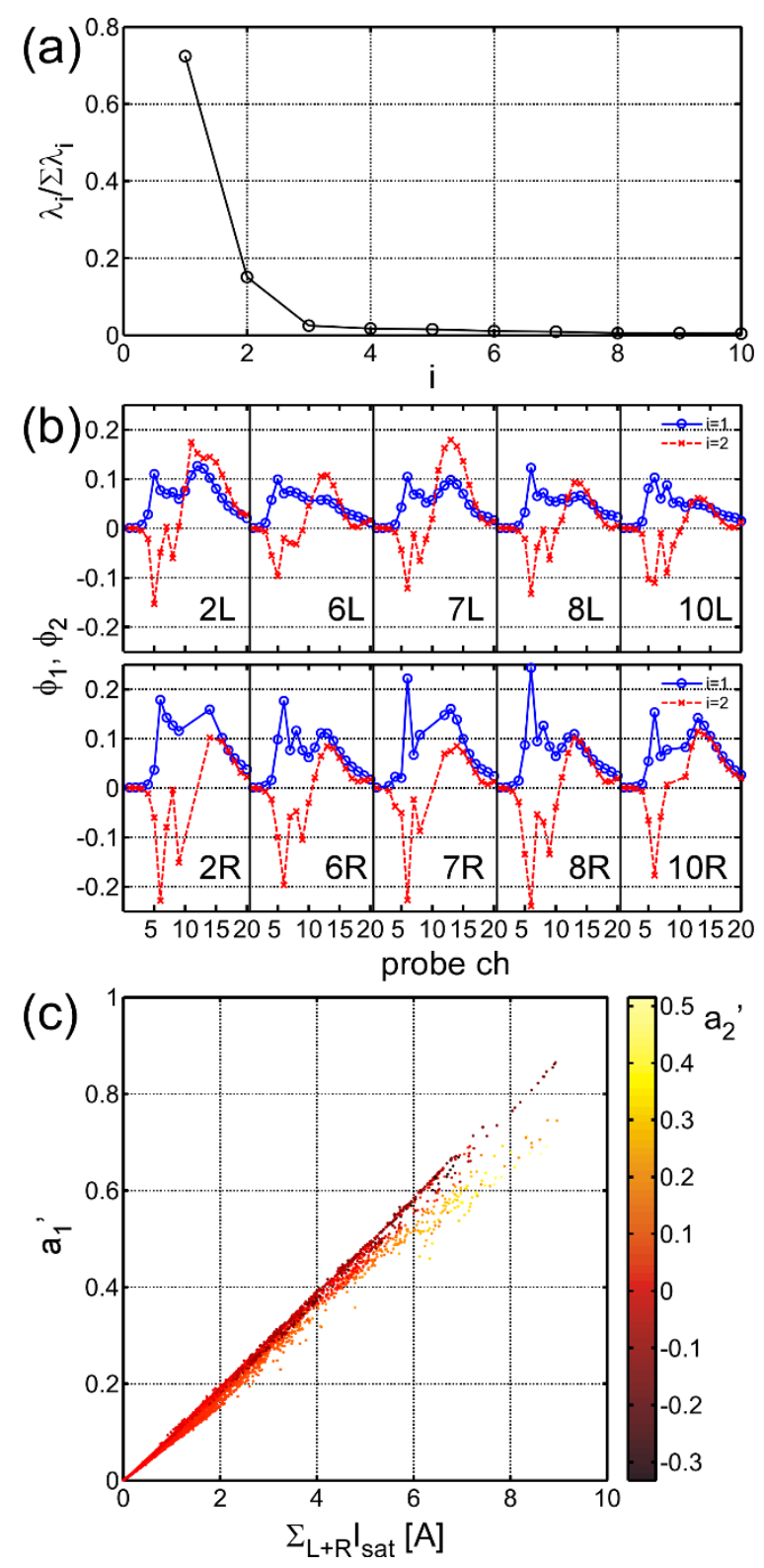

Fig. 3 (Color online) (a) $\lambda_{i} / \sum_{k=1}^{n} \lambda_{k}$ as a function of $i$. (b) $\phi_{1}$ (solid line with circle) and $\phi_{2}$ (dashed line with cross) profiles as a function of the probe channel on each divertor plate. (c) Scatter plot of $a_{1}^{\prime}$ versus $\sum_{L+R} I_{\text {sat }}$. Dot color indicates the $a_{2}^{\prime}$ value. 


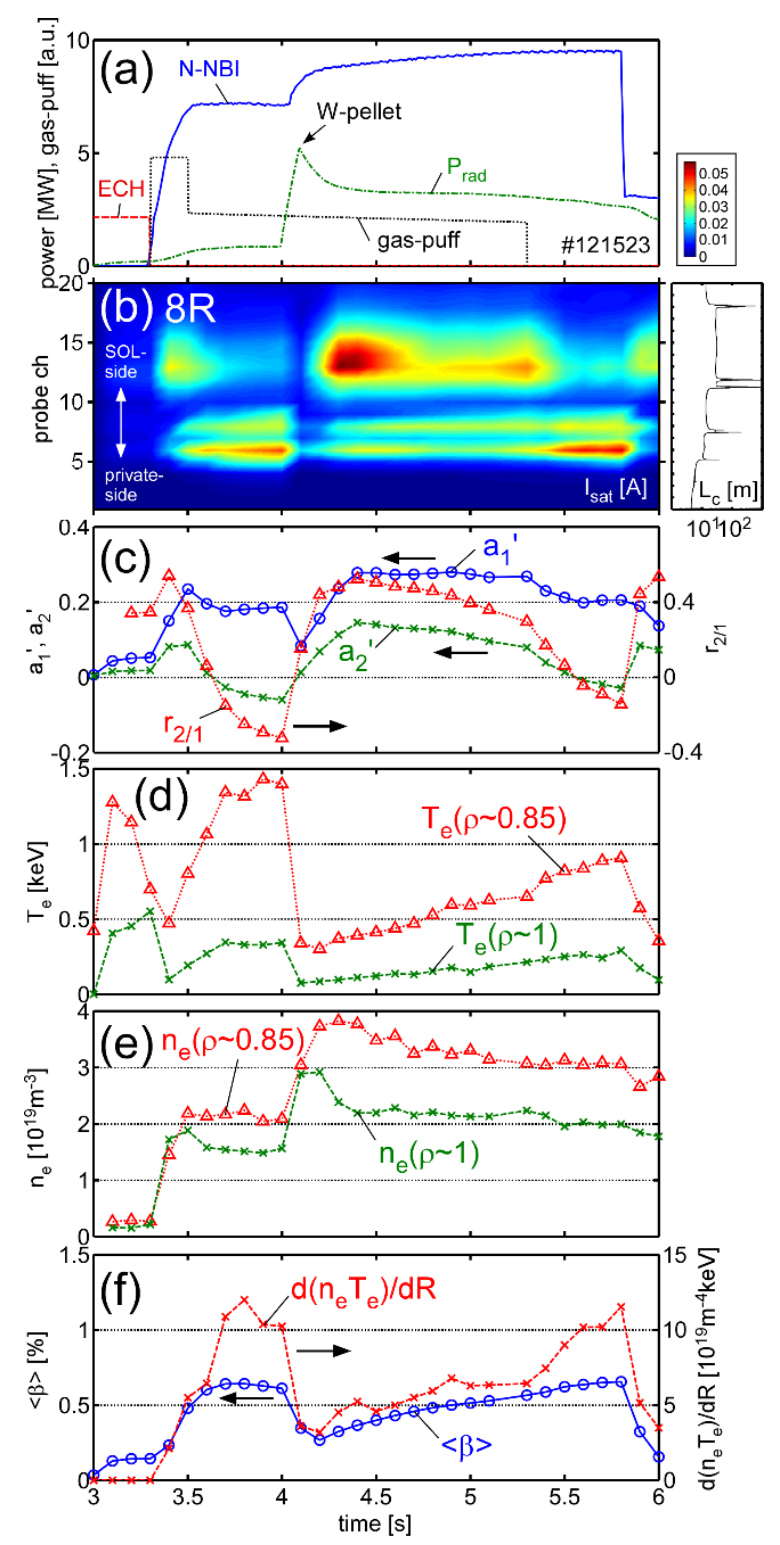

Fig. 4 (Color online) (a) Time trends of input powers of N-NBI (solid line) and ECH (dashed line), $P_{\text {rad }}$ (dashed dotted line), and gas-puff signal (dotted line) in a discharge. (b) Contour plot of $I_{\text {sat }}$ on $8 \mathrm{R}$ plate and $L_{\mathrm{c}}$ profile. (c) Time series of $a_{1}^{\prime}$ (solid line with circle), $a_{2}^{\prime}$ (dashed line with cross), $r_{2 / 1}$ (dotted line with triangle), (d) $T_{\mathrm{e}}$ at $\rho \sim 1$ (dashed line with cross) and 0.85 (dotted line with triangle), (e) $n_{\mathrm{e}}$ at $\rho \sim 1$ (dashed line with cross) and 0.85 (dotted line with triangle), and (f) $\langle\beta\rangle$ (solid line with circle), and $\mathrm{d}\left(n_{\mathrm{e}} T_{\mathrm{e}}\right) / \mathrm{d} R$ at $\rho \sim[0.85,0.95]$ (dashed line with cross). 

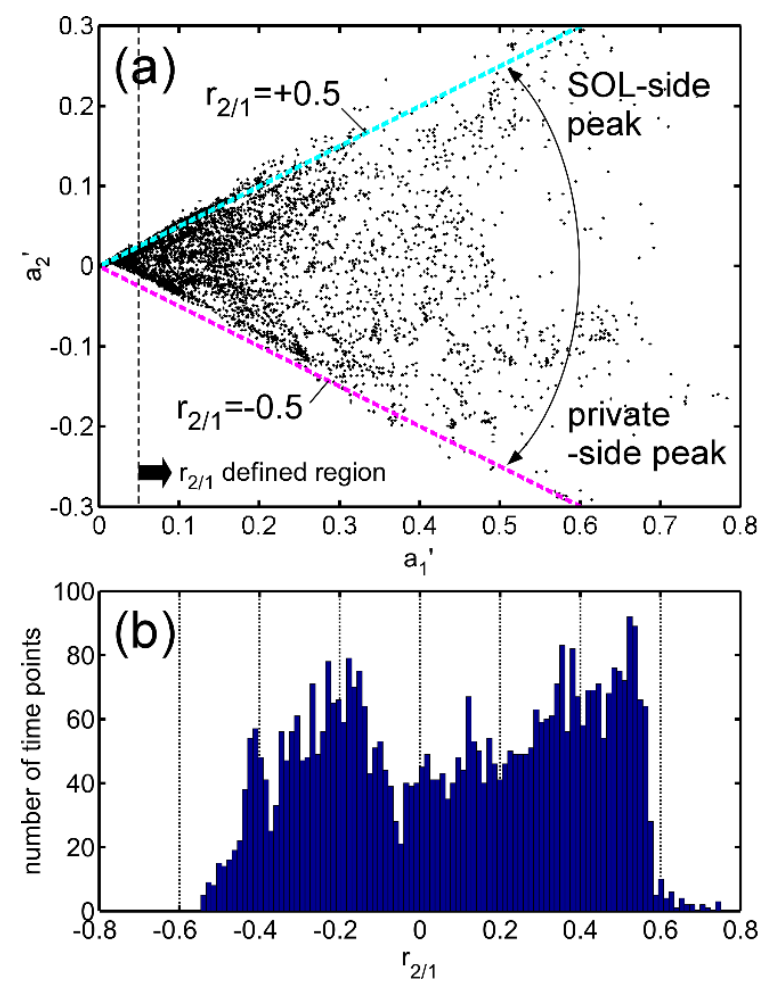

Fig. 5 (Color online) (a) Scatter plot of $a_{2}^{\prime}$ versus $a_{1}^{\prime}$. Oblique dashed lines indicate $r_{2 / 1}=-0.5$ and 0.5 . Vertical dashed line indicates $a_{1}^{\prime}=0.05$ position. (b) Histogram of $r_{2 / 1}(t)$.

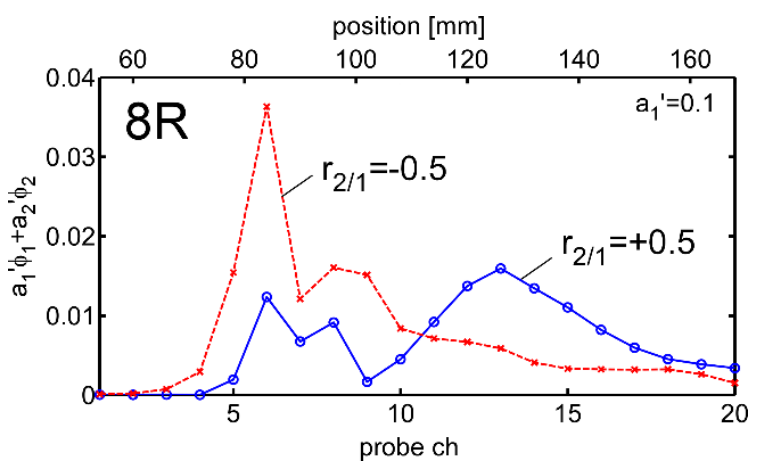

Fig. 6 (Color online) Reconstructed footprint profiles on $8 \mathrm{R}$ plate from $a_{1}^{\prime} \phi_{1}+a_{2}^{\prime} \phi_{2}$ with $r_{2 / 1}=-0.5$ and +0.5 . 


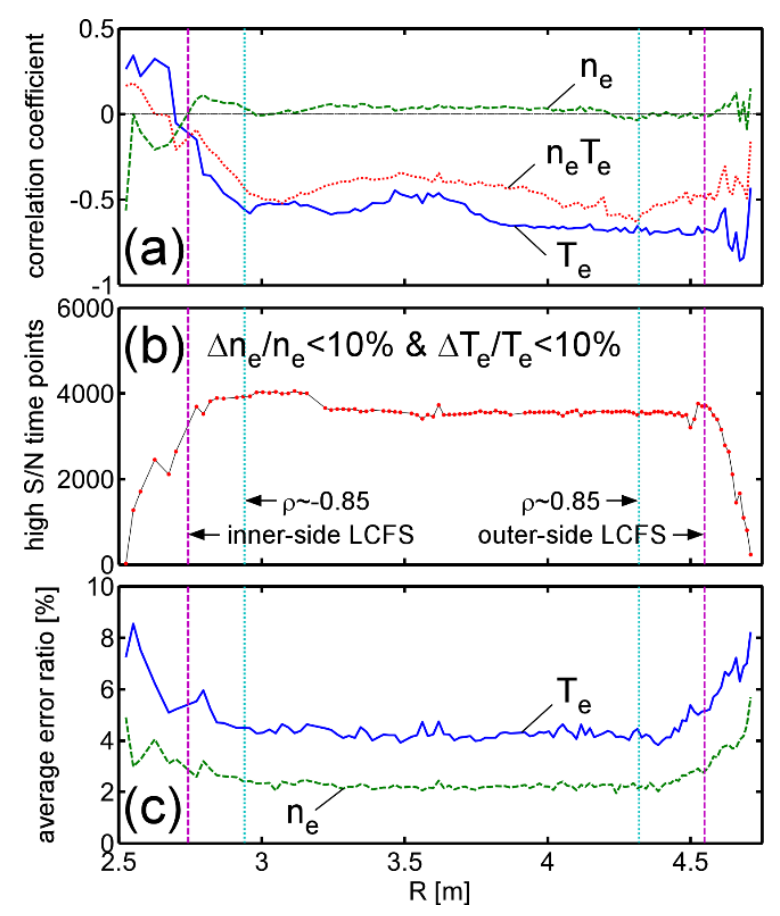

Fig. 7 (Color online) (a) Cross-correlation coefficients $R\left(r_{2 / 1}, T_{\mathrm{e}}\right)$ (solid line), $R\left(r_{2 / 1}, n_{\mathrm{e}}\right)$ (dashed line), and $R\left(r_{2 / 1}, n_{\mathrm{e}} T_{\mathrm{e}}\right)$ (dotted line) as a function of the major radius $R$. Analyzed high $\mathrm{S} / \mathrm{N}$ time points with $\Delta T_{\mathrm{e}} / T_{\mathrm{e}}<10 \%$ and $\Delta n_{\mathrm{e}} / n_{\mathrm{e}}<10 \%$. (c) Mean $\Delta T_{\mathrm{e}} / T_{\mathrm{e}}$ and $\Delta n_{\mathrm{e}} / n_{\mathrm{e}}$ of analyzed time points. Vertical dashed and dotted lines indicate $|\rho| \sim 1$ and 0.85 , respectively. 

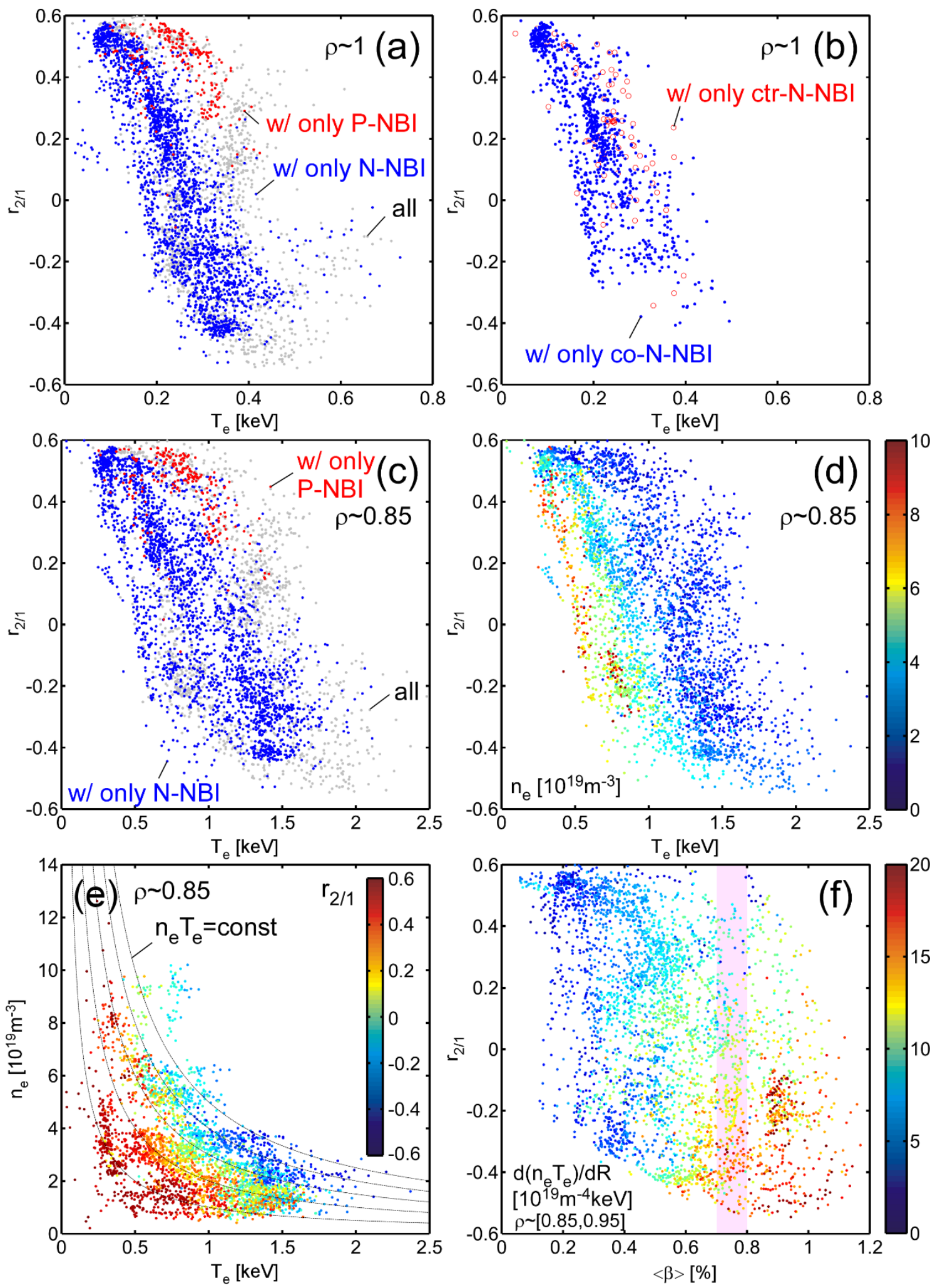

Fig. 8 (Color) (a) $r_{2 / 1}$ versus $T_{\mathrm{e}}$ at $\rho \sim 1$, where dots with only N-NBI and P-NBI are colored in blue and red, respectively. (b) Similar plot with only co-injected (blue dot) and ctr-injected NBs (red circle). (c) $r_{2 / 1}$ versus $T_{\mathrm{e}}$ at $\rho \sim 0.85$, where dots with only N-NBI and P-NBI are colored in blue and red, respectively. (d) Similar plot with dots colored by $n_{\mathrm{e}}$. (e) $n_{\mathrm{e}}$ versus $T_{\mathrm{e}}$ at $\rho \sim 0.85$ colored by $r_{2 / 1}$. (f) $r_{2 / 1}$ versus $\langle\beta\rangle$ colored by $\mathrm{d}\left(n_{\mathrm{e}} T_{\mathrm{e}}\right) / \mathrm{d} R$ at $\rho \sim[0.85,0.95]$. Hatched area indicates $0.7 \%<\langle\beta\rangle<0.8 \%$. 


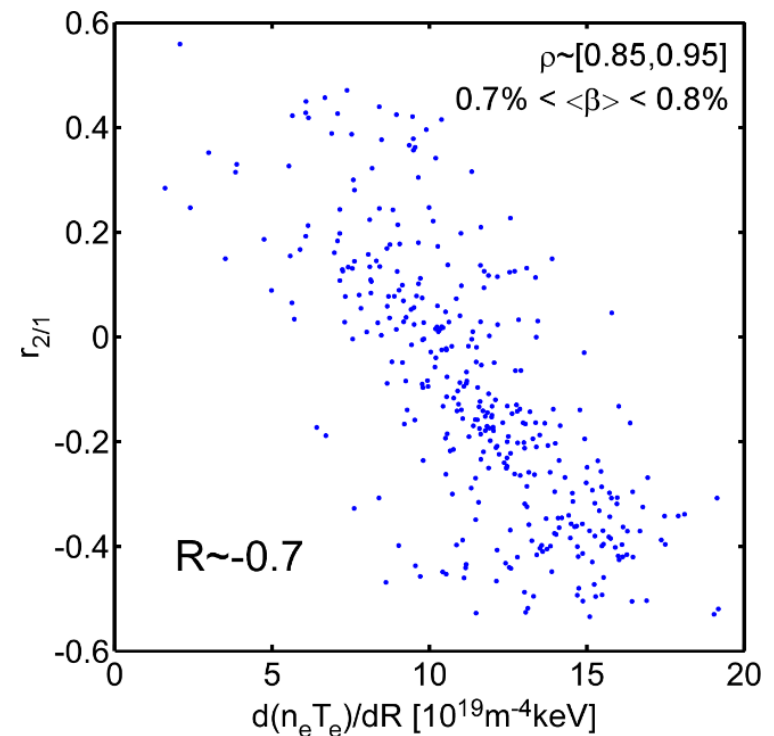

Fig. 9 (Color online) $r_{2 / 1}$ versus $\mathrm{d}\left(n_{\mathrm{e}} T_{\mathrm{e}}\right) / \mathrm{d} R$ at $\rho \sim[0.85,0.95]$ and $0.7 \%<\langle\beta\rangle<0.8 \%$. 